\title{
Bibliographie de Dominique Buchillet
}

Laure Emperaire et Lúcia Hussak van Velthem

\section{(2) OpenEdition}

Journals

Édition électronique

URL : https://journals.openedition.org/jsa/17505

DOI : 10.4000/jsa. 17505

ISSN : 1957-7842

Éditeur

Société des américanistes

Édition imprimée

Date de publication : 20 décembre 2019

Pagination : 211-216

ISSN : 0037-9174

\section{Référence électronique}

Laure Emperaire et Lúcia Hussak van Velthem, «Bibliographie de Dominique Buchillet », Journal de la Société des américanistes [En ligne], 105-2 | 2019, mis en ligne le 20 décembre 2019, consulté le 04 septembre 2022. URL : http://journals.openedition.org/jsa/17505 ; DOI : https://doi.org/10.4000/jsa. 17505 


\title{
Bibliographie de Dominique Buchillet
}

\author{
Établie par Laure EMPERAIRE \\ et Lúcia HussaK van Velthem*
}

\section{Thèse et livres édités}

\section{Buchillet Dominique}

1983 Maladie et mémoire des origines chez les Desana du Uaupés (Brésil), thèse de doctorat en ethnologie, université Paris X, Nanterre.

2007 Bibliografia crítica da saúde indígena no Brasil 1844-2006, Abya Yala, Quito (http://horizon.documentation.ird.fr/exl-doc/pleins_textes/ divers14-09/010041779.pdf, consulté le 10/12/19).

Buchillet Dominique (dir.)

1991 Medicinas tradicionais e medicina ocidental na Amazônia [contribuições científicas apresentadas no Encontro de Belém - 17/novembro a 1\% dezembro de 1989], MPEG/CNPq/SCT/CEJUP/UEP, Belém.

\section{Organisation et édition commentée de recueils de récits amérindiens}

Barbosa Manuel Marcos, Adriano Manuel Garcia, Pedro Garcia, Benjamin Garcia et Dominique Buchillet

2000 Upíperi kalísi. Histórias de antigamente: histórias dos antigos TaliaseriPhukurana (versão do clã Kabana-idakena-yanapere), UNIRVA, Iauareté/ FOIRN, São Gabriel da Cachoeira, 288 p.

Diakuru, KISIBI (narrateurs) et Dominique BuchiLlet

1996 A mitologia sagrada dos antigos Desana do grupo Wari dihputiro põrã, narrateurs Américo Castro Fernandes (Diakuru) et Durvalino Moura Fernandes (Kisibi), UNIRT, Cucura do Igarapé Cucura (Amazonas, Brasil)/FOIRN (Coleção Narradores indígenas do Rio Negro, 2), São Gabriel da Cachoeira (Amazonas, Brasil)/ORSTOM, Paris, 196 p.

2006 Bueri Kãdiri Marĩriye. Os ensinamentos que não se esquecem, FOIRN (Coleção Narradores Indígenas do Rio Negro, 8), São Gabriel da Cachoeira (Amazonas, Brasil)/UNIRT, Santo Antonio (Amazonas, Brasil), 168 p.

* L. Emperaire: Directrice de recherche, IRD [laure.emperaire@ird.fr]; L. Hussak van Velthem: Museu Paraense Emilio Goeldi (MPEG) - Ministério da Ciência, Tecnologia, Inovações e Comunicações (MCTIC) [luciavelthem@museu-goeldi.br]. 
Galvão Wenceslau Sampaio, Raimondo Castro Galvão (narrateurs) et Dominique BuCHILlET 2004 Livro dos antigos Desana-Guahari Diputiro Porã, FOIRN (Coleção Narradores Indígenas do Rio Negro, 7), São Gabriel da Cachoeira (Amazonas, Brasil)/ ONIMRP, Comunidade do Pato no médio rio Papuri (Amazonas, Brasil), 687 p.

\section{Articles et chapitres de livres}

\section{Buchillet Dominique}

1986a «Droits territoriaux, raisons de sécurité nationale et politique indigéniste au Brésil », Journal de la Société des américanistes, 72, p. 230-236.

1986 b «Entreprises minières et Indiens dans le haut Rio Negro », Journal de la Société des américanistes, 72, p. 236-240.

1987a «La politique indigéniste de la Nouvelle République » Journal de la Société des américanistes, 73, p. 264-277.

1987 b «Les Indiens Tukano et l'exploitation minière de leur territoire », Journal de la Société des américaniste, 73, p. 277-280.

1987 " "Personne n'est là pour écouter." Les conditions de mise en forme des incantations thérapeutiques chez les Desana du Uaupès brésilien », Amerindia, 12 , p. 7-32.

1988a « Interpretação da doença e simbolismo ecológico entre os índios Desana », Boletim do Museu Parense Emílio Goeldi, 4 (1), p. 27-42.

1988 b «La question des droits indigènes au Brésil », Journal de la Société des américanistes, 74, p. 195-205.

1988c «Les Indiens Tukano et les colonies indigènes », Journal de la Société des américanistes, 74, p. 206-207.

1990a «Los poderes del hablar. Terapia y agresión entre los índios Desana del Vaupés brasileiro ", in Ellen Basso et Joel Sherzer (dir.), Las culturas nativas latinoamericanas a través de su discurso, Abya Yala, Quito/MLAL (500 Años, 24), Roma, p. 319-354.

1990b «Agressão aos índios Tukano », Informativo INESC, 4 (15 jun.), p. 7-7.

1990c «Os povos Tukano orientais e a questão da saúde », Amazonas em Tempo, Caderno Cultura, Manaus, 29 jan., p. 4-5.

1990d «Situación de los Tukano », Boletin del IWGIA, 6, p. 52-61.

1990e «Pari-Cachoeira: le laboratoire Tukano du Projet Calha Norte », Ethnies. Droits de l'Homme et peuples autochtones, 11-12, p. 128-135.

1991a «A antropologia da doença e os sistemas oficiais de saúde », in Dominique Buchillet (dir.), Medicinas tradicionais e medicina ocidental na Amazônia [contribuições científicas apresentadas no Encontro de Belém - 17/novembro a 1\% dezembro de 1989], MPEG/CNPq/SCT/CEJUP/UEP, Belém, p. 21-44.

$1991 b$ « Introdução » de « Representações e práticas das medicinas tradicionais », in Dominique Buchillet (dir.), Medicinas tradicionais e medicina ocidental na Amazônia [contribuições científicas apresentadas no Encontro de Belém - 17/novembro a 1\% dezembro de 1989], MPEG/CNPq/SCT/CEJUP/UEP, Belém, p. 63-64.

1991c 《Introdução » de « Impacto do contato sobre as representações tradicionais da doença e de seu tratamento », in Dominique Buchillet (dir.), Medicinas 
tradicionais e medicina ocidental na Amazonia [contribuições científicas apresentadas no Encontro de Belém - 17/novembro a 1\% dezembro de 1989], MPEG/CNPq/SCT/CEJUP/UEP, Belém, p. 161-173.

1991d 《Introdução » de « A questão da integração dos sistemas médicos, problema e perspectivas », in Dominique Buchillet (dir.), Medicinas tradicionais e medicina ocidental na Amazônia [contribuições científicas apresentadas no Encontro de Belém - 17/novembro a 1\% dezembro de 1989], MPEG/CNPq/ SCT/CEJUP/UEP, Belém, p. 243-246.

1991e «Pari Cachoeira: o laboratório tukano do projeto Calha Norte », in Carlos A. Ricardo (dir.), Povos indígenas no Brasil. 1987/88/89/90, Centro Ecumênico de Documentação e Informação (Aconteceu especial, 18), São Paulo, p. 107-115 (https://horizon.documentation.ird.fr/exl-doc/pleins_textes/pleins_textes_6/b_ fdi_33-34/38979.pdf, consulté le 10/12/19).

1992 « Nobody is there to hear. Desana therapeutic incantations », in E. Jean Langdon et Gerhard Baer (dir.), Portals of power: shamanism in South America, University of New Mexico Press, Albuquerque, p. 211-230.

1993 «Droits constitutionnels et démarcation des terres au Brésil », Journal de la Société des américanistes, 79, p. 225-231.

1994a «Droits indigènes, militarisation et violence contre les Indiens au Brésil », Journal de la Société des américanistes, 80, p. 243-250.

1994b « Prefácio », in Alcionílio Brüzzi Alves da Silva, Crenças e lendas do Uaupés, presentación de Walter Ivan de Azevedo, Abya Yala, Quito/CEDEM, Manaus, p. 5-19 (https://horizon.documentation.ird.fr/exl-doc/pleins_textes/ divers11-10/010020940.pdf, consulté le 10/12/19).

1995a «Perles de verre, parures de blancs et "pots de paludisme", épidémiologie et représentations Desana des maladies infectieuses (haut Rio Negro, Brésil) », Journal de la Société des américanistes, 81, p. 181-206.

1995 b «Contas de vidro, enfeites de branco e "potes de malária": epidemiologia e representações de doenças infecciosas entre os Desana », documents d'archives, Departamento de Antropologia, Universidade de Brasília (Série Antropologia, 187), Brasília, p. 1-24 (https://horizon.documentation.ird.fr/exl-doc/pleins_textes/pleins_textes_7/b_fdi_51-52/010019241.pdf, consulté le 10/12/19).

1995c «Les vieux démons de la politique indigéniste au Brésil », Journal de la Société des américanistes, 81, p. 267-274.

1996a «Épidémies et médecines traditionnelles en Amazonie brésilienne », Orstom Actualités, 50, p. 2-8.

1996b «Le décret 1775/96: une nouvelle forme de spoliation des territoires indigènes? », Journal de la Société des américanistes, 82, p. 341-347.

1997a « De la colonie à la République: images de l'Indien, politique et législation indigénistes au Brésil », Cahiers des Amériques latines, 23, p. 73-93.

1997 « Les conséquences du décret présidentiel 1775/96 pour les droits territoriaux des Indiens ", Journal de la Société des américanistes, 83, p. 295-300.

1997c «Tuberculose, busca de assistência médica e observância terapêutica na Amazônia brasileira », França-Flash Saúde, 11, p. 1-8.

1997d « Tuberculose no alto Rio Negro », Parabólicas, 27 (4), p. 9. 
1998 «Les nouvelles menaces contre les droits indigènes au Brésil », Journal de la Société des américanistes, 84-1, p. 237-248.

1999 «Une lueur d'espoir à l'aube des commémorations de l'an 2000 ? », Journal de la Société des américanistes, 85, p. 399-409.

2000a «Tuberculose, cultura e saúde pública », documents d'archives, Departamento de Antropologia, Universidade de Brasília (Série Antropologia, 273), Brasília, p. 1-17 (http://dan.unb.br/images/doc/Serie273empdf.pdf, consulté le 10/12/19).

2000b « Apresentação », in Manuel Marcos Barbosa, Adriano Manuel Garcia, Pedro Garcia, Benjamin Garcia et Dominique Buchillet, Upíperi kalísi. Histórias de antigamente: histórias dos antigos Taliaseri-Phukurana (versão do clã Kabana-idakena-yanapere), Unirva, Iauareté/FOIRN, São Gabriel da Cachoeira, p. 9-15.

2001a «Tuberculose et santé publique: les multiples facteurs impliqués dans l'adhésion au traitement », Autrepart, 19, p. 71-90.

2001 b «esafios para a implantação do DSEI-RN », in Carlos A. Ricardo (dir.), Povos indígenas no Brasil 1996-2000, Instituto Socioambiental, São Paulo, p. 255-257.

2001c «Tuberculose, antropologia e saúde pública », in Anais do seminário sobre alcoolismo e vulnerabilidade às DST/AIDS entre os povos indígenas da Macrorregião Sul, Sudeste e Mato Grosso do Sul [Londrina, 1999], Ministério da Saúde/Secretaria de Políticas de Saúde/Coordenação de DST e AIDS, Brasília, p. 61-76.

2002a «Droits constitutionnels, ressources génétiques, protection du patrimoine génétique et des savoirs traditionnels des populations indigènes », Journal de la Société des américanistes, 88, p. 245-260.

2002 b «Contas de vidro, enfeites de branco e "potes de malaria": epidemiologia e representações de doenças infecciosas entre os Desana do alto Rio Negro ",, in Bruce Albert et Alcida R. Ramos (dir.), Pacificando o branco: cosmologias do contato no norte-Amazônico, IRD/UNESP, São Paulo, p. 113-142.

2003 «Contact interethnique et épidémies au Brésil », Journal international de bioéthique, 14 (1-2), p. 83-99.

2004 «Sorcery beliefs, transmission of shamanic knowledge and therapeutic practice among the Desana of the upper Rio Negro region (Brazil) », in Neil L. Whitehead and Robin B. Wright (dir.), In darkness and secrecy. The anthropology of assault sorcery in Amazônia, Duke University Press, Durham (NC), p. 109-131.

2013 «Mythology, shamanism and epidemic diseases: a view from the upper Rio Negro region », in Patience Epps et Kristine Stenzel (dir.), Upper Rio Negro. Cultural and linguistic interaction in northwestern Amazonia, Museu do Índio/ Funai/Museu Nacional, Rio de Janeiro, p. 441-474.

2016 «Épidémies, guerres et esclavage: les causes de la dépopulation indigène au Brésil », in Philippe Erikson (dir.), Trophées : études ethnologiques, indigénistes et amazonistes offertes à Patrick Menget, vol. 2. Guerre, chamanisme et rencontres interethniques, Société d'Ethnologie, Nanterre, p. 39-55.

2018 «Colonization and epidemic diseases in the upper Rio Negro region, Brazilian Amazon (Eighteenth-Nineteenth centuries) », Boletin de Antropologia, 33 (55), p. 102-122. 


\title{
Articles et chapitres collectifs
}

\author{
Buchillet Dominique et Pierre Gazin \\ 1998 «A situação da tuberculose na população indígena do alto Rio Negro (Estado \\ do Amazonas, Brasil) », Cadernos de Saúde Pública, 14 (1), p. 181-185. \\ Buchillet Dominique, Robin Wright, Márcio Meira, Carlos A. Ricardo et Aloisio \\ Cabalzar Filho \\ 1998 Contribution au chapitre « A história a partir da chegada dos europeus », in \\ Aloisio Cabalzar Filho et Carlos A. Ricardo (dir.), Povos indígenas do alto \\ e médio rio Negro: uma introdução à diversidade cultural e ambiental do \\ noroeste da Amazônia brasileira, ISA, São Paulo/FOIRN, São Gabriel da \\ Cachoeira, p. 73-124.
}

Buchillet Dominique, Geraldo Andrello et Eduardo Neves

1998 Contribution au chapitre « O ambiente natural e a ocupação tradicional dos povos indígenas », in Aloisio Cabalzar Filho et Carlos A. Ricardo (dir.), Povos indígenas do alto e médio rio Negro: uma introdução à diversidade cultural e ambiental do noroeste da Amazônia brasileira, ISA, São Paulo/FOIRN, São Gabriel da Cachoeira, p. 55-72.

Garnelo Luiza et Dominique Buchillet

2006 «Taxonomias das doenças entre os índios Baniwa (arawak) e Desana (tukano oriental) do alto rio Negro (Brasil) », Horizontes Antropológicos, 12 , p. $231-260$.

Gomez Elsa et Dominique Buchillet

1986 « Propuesta para una grafia Tukano normalizada », Chantiers Amerindia, 11, p. 1-36 ; trad. « Proposta para uma grafia Tukano normalizada », Chantiers Amerindia, 11 (suppl. 3b), p. 1-10.

\section{Rapports techniques}

Buchillet Dominique

1990 Os índios da região do Alto Rio Negro: história, etnografia e situação das terras, Laudo antropológico redigido para a Procuradoria Geral da República na ação visando a demarcação sob forma única da região do Alto Rio Negro, Brasília (revisado e ampliado em 1997), 90 p.

1992 Território, ecologia, sistema produtivo indígena e organização social no alto Rio Negro, Brasília, ms, 10 p.

1998a As medicinas tradicionais e sua articulação com os sistemas oficiais de saúde, IRD/ISA, São Paulo, 11 p.

1998b Fatores sócio-culturais e econômicos de risco para HIV e Tuberculose na região do alto Rio Negro (Amazônia Brasileira), Relatório n ${ }^{\circ} 1 \mathrm{CNPq} /$ ORSTOM n ${ }^{\circ}$ 910213/97-6.

1998c Levantamento e avaliação de projetos de saúde em áreas indígenas da Amazônia legal, Suporte a projetos de saúde culturalmente sensíveis, Relatório de consultoria para o PPTAL/GTZ/Banco Mundial, $71 \mathrm{p}$.

1998d Tuberculose e saúde pública: o papel do antropólogo nos programas nacionais de controle e de luta contra a tuberculose, IRD/ISA, São Paulo, 13 p. 


\section{Comptes rendus}

\section{BuChillet Dominique}

1984 «Makuna Social Organization. A Study in Descent, Alliance and the Formation of Corporate Groups de K. Arhem (Uppsala, Almsqvist and Wiksell International, 1981) », L'Homme, 24 (3-4), p. 145-146.

1985 «The Fish People. Linguistic Exogamy and Tukanoan Identity in North-west Amazonia de J. Jackson (Cambridge, Cambridge University Press, 1983) », L'Homme, 25 (2), p. 169-170.

1990 Manipulando identidades no médio Solimões. Análise crítica de « O Navio Encantado. Etnia e Alianças em Tefé de P. Faulhaber (Belém, MCT/CNPq, 1987, 253 p.) », Anuário Antropológico, 87, p. 259-267.

1995a «Médecins et chamanes des Andes de F.L. Blanc (L’Harmattan, Paris, 1995, 206 p.) », Journal de la Société des américanistes, 81, p. 361-363.

1995 « Saúde \& Povos indígenas de R.V. Santos \& C.E.A. Coimbra Jr., orgs (Rio de Janeiro, Editora Fiocruz, 1994, 251 p.) », Cadernos de Saúde Pública, 11 (2), p. 338-341.

1996 «La civilisation amérindienne de C.R. Cros (Paris, PUF, 1995, 127 p.)», Journal de la Société des américanistes, 82, p. 398-400.

1997 «Chamanismo en latinoamérica: una revisión conceptual de I. Lagarriga, J. Galinier \& M. Persin, eds (Mexico, Plaza y Valdès Editores, 1997, 244 p.) », Journal de la Société des américanistes, 83, p. 348-350.

1998a «Mindful of Famine. Religious Climatology of the Warao Indians de J. Wilbert (Cambridge, Harvard University Press, 1996, 363 p.) », Journal de la Société des américanistes, 84 (1), p. 340-343.

1998 b «L'Indigénisme de H. Favre (Paris, PUF, 1996, n. 3088, 128 p.) », Journal de la Société des américanistes, 84 (1), p. 348-350.

1999a «Nukak de G.G. Politis (Bogota, SINCHI, 1998, 426 p.) », Journal de la Société des américanistes, 85, p. 481-483.

1999b «History, Power, and Identity. Ethnogenesis in the Americas 1492-1992 de J.D. Hill, ed. (Iowa City, University of Iowa Press, 1996, 277 p.) », Journal de la Société des américanistes, 85, p. 447-448.

1999c «Del microscopio a la maraca de J. Chiappino \& C. Alès, dir. (Caracas, Editorial Ex-Libris, 1997, 400 p.) », Journal de la Société des américanistes, 85, p. 486-488.

1999d «O Dono dos Sonhos de S.L.R. Medeiros (São Paulo, Razão Social Empreendimentos Editoriais Ltda., 1991, 184 p.) », Journal de la Société des américanistes, 85, p. 484-486.

1999e «Religion and Identity in the Americas. Anthropological Perspectives from Colonial Times to the Present de B. Hülsewiede \& I.W. Shröder, eds (Bonner Akademische Studien Part I, vol. 28, 1997, 184 p.) », Journal de la Société des américanistes, 85, p. 445-447.

1999f «Antropologia da Saúde. Traçando Identidade e Explorando Fronteiras de P.C. Alves \& M.C. Rabelo, orgs (Rio de Janeiro, Relume \& Dumará, 1998, 245 p.) », Cadernos de Saúde Pública, 1999 (15), p. 911-912. 